\title{
RESEARCH
}

Open Access

\section{Iron accentuated reactive oxygen species release by NADPH oxidase in activated microglia contributes to oxidative stress in vitro}

Young J. Yauger ${ }^{1}$, Sara Bermudez ${ }^{2}$, Kasey E. Moritz ${ }^{1}$, Ethan Glaser ${ }^{3}$, Bogdan Stoica ${ }^{3}$ and Kimberly R. Byrnes ${ }^{1,2^{*}}$ (D)

\begin{abstract}
Background: Excessive iron contributes to oxidative stress after central nervous system injury. NADPH oxidase (NOX) enzymes are upregulated in microglia after pro-inflammatory activation and contribute to oxidative stress. The relationship between iron, microglia, NOX, and oxidative stress is currently unclear.

Methods: We evaluated the effects of iron on lipopolysaccharide (LPS)-activated microglia and its secondary effect within neuronal co-cultures. Further, NOX2 and four specific inhibitors were tested to evaluate the relationship with the reactive oxygen species (ROS)-producing enzymes.

Results: An iron dose-dependent increase in ROS production among microglia treated with LPS was identified. Interestingly, despite this increase in ROS, inflammatory polarization alterations were not detected among the microglia after exposure to iron and LPS. Co-culture experimentation between primary neurons and exposed microglia (iron and LPS) significantly reduced neuronal cell number at $24 \mathrm{~h}$, suggesting a profound neurotoxic effect despite the lack of a change in polarization phenotype. NOX2 and NOX4 inhibition significantly reduced ROS production among microglia exposed to iron and LPS and reduced neuronal damage and death in response to microglial co-culture.

Conclusions: In conclusion, iron significantly increased ROS production and neurotoxicity without exacerbating LP-activated microglia phenotype in vitro, suggesting that iron contributes to microglia-related oxidative stress, and this may be a viable therapeutic target for injury or neurodegeneration. Further, this study highlights both NOX2 and NOX4 as potential therapeutic targets in the treatment of iron-induced microgliarelated inflammation and neurotoxicity.
\end{abstract}

Keywords: BV2, Iron sulfate, Neuronal toxicity, Microglial polarization, Neuroinflammation

\footnotetext{
*Correspondence: kimberly.byrnes@usuhs.edu

${ }^{1}$ Neuroscience Graduate Program, Uniformed Services University of the Health Sciences, Room C2099, 4301 Jones Bridge Road, Bethesda, MD 20814, USA

${ }^{2}$ Department of Anatomy, Physiology, and Genetics, Uniformed Services University of the Health Sciences, Room C2099, 4301 Jones Bridge Road, Bethesda, MD 20814, USA

Full list of author information is available at the end of the article
}

(c) The Author(s). 2019 Open Access This article is distributed under the terms of the Creative Commons Attribution 4.0 International License (http://creativecommons.org/licenses/by/4.0/), which permits unrestricted use, distribution, and reproduction in any medium, provided you give appropriate credit to the original author(s) and the source, provide a link to the Creative Commons license, and indicate if changes were made. The Creative Commons Public Domain Dedication waiver (http://creativecommons.org/publicdomain/zero/1.0/) applies to the data made available in this article, unless otherwise stated. 


\section{Background}

Injury to the central nervous system (CNS), such as a traumatic brain injury (TBI), results in secondary pathophysiology that includes edema, glutamate toxicity, and inflammation. Neuro-inflammation begins within the focal lesion site acutely after injury and can persist chronically [1-4]. A hallmark sign of neuroinflammation is post-injury oxidative stress. Multiple reports of oxidative damage to neurons through carbonylation, nitrosylation, lipid peroxidation, and DNA damage have been observed acutely and chronically in TBI [5-8].

A major contributor to oxidative stress in the $\mathrm{CNS}$ are microglia, the parenchymal immune cell of the brain. Microglia can modulate the inflammatory response through cytokines, chemokines, and reactive oxygen species (ROS) [9]. Microglia are known to assume a pro-inflammatory phenotype shortly after injury and can persist for years $[2,10]$. Originally described as the respiratory burst, superoxide generation by microglia is an effective modality to mitigate pathogens or other perturbations in the brain $[11,12]$. This mechanism is mediated through the activation of the NADPH oxidase 2 (NOX2) enzyme. NOX2 is a member of a family of enzymes consisting of seven isoforms responsible for producing various ROS. Of these family members, two isoforms, NOX2 and NOX4, are present and elevated within microglia after TBI [13]. Although they are isoforms of the same family of enzymes, NOX2 and NOX4 possess significant disparities. NOX2 requires phosphorylation to become activated, followed by multi-subunit assembly before superoxide synthesis can begin. Contrastingly, NOX4 is constitutively active and produces hydrogen peroxide for various cellular activities throughout the cell to maintain homeostasis [14].

Another source of oxidative stress within TBI is iron. Iron is elevated within TBI lesions, particularly among moderate to severe injuries $[15,16]$. Under steady state conditions, iron is transported within transferrin and actively transported across the cell membrane where it is subsequently sequestered within ferritin (FER) to protect the cell from its unique physical properties [17]. Excessive extracellular $\mathrm{Fe}^{2+}$ not sequestered within proteins is instead transported via divalent metal transporter 1 (DMT1) and delivered to the intracellular compartment $[18,19]$. Once in the intracellular compartment, $\mathrm{Fe}^{2+}$ is immediately sequestered within FER. If FER reaches its capacity for iron, the remaining unbound iron may undergo a spontaneous non-enzymatic Fenton, Haber-Weiss reaction [20]. Specifically, this reaction requires hydrogen peroxide to oxidize iron to its stable $\mathrm{Fe}^{3+}$ form. Following, it can reduce back into $\mathrm{Fe}^{2+}[21]$. More concerning, this non-enzymatic reaction results in the synthesis of hydroxyl radicals, the most reactive form of ROS, which has been implicated as the major destructive ROS within TBI [22-24].
It is well established that iron deposition, oxidative stress, and activated microglia are present within CNS lesions. This triad is considered a major contributor to neural degeneration among many CNS diseases. The exact interactions between the components of the triad are unknown; however, multiple studies purport iron chelation or antioxidant therapies can reduce CNS inflammation leading to improved functional outcomes, reduced lesion volumes, and altered neuronal morphology after injury [25-27]. Since it is generally accepted that improved neuronal survivability following injury may translate into improved long-term outcomes, it is imperative to evaluate the relationship between $\mathrm{Fe}^{2+}$, oxidative stress, microglial polarization, and their effect on neuronal survivability. Therefore, we hypothesized that $\mathrm{Fe}^{2+}$ can accentuate a ROS-producing pro-inflammatory phenotype in activated microglia, including primary cells or microglial cell lines, in a NOX2- and/or a NOX4-dependent manner, which subsequently reduces neuronal viability in vitro. This experiment involved both primary microglial cells and the BV2 microglial cell line. While the microglial cell line BV2 has been shown to have some marked differences from primary cell line responses [28], it has noted to be an acceptable alternative or adjunct to primary cells in many experimental lines of investigation [29-31], and we herein demonstrate that it responds similarly to primary cells for all outcomes evaluated.

\section{Methods \\ Cell culture}

BV2 microglia cell cultures (a gift from Dr. Carol Colton) were maintained with Dulbecco's modified Eagle medium (Gibco, Gaithersburg, MD), 1\% L-glutamine (Gibco), 1\% sodium pyruvate (Gibco), 10\% fetal bovine serum (FBS) (Hyclone, Logan, UT), and 1\% penicillin and streptomycin (Thermo Fisher Scientific, Pittsburgh, PA) [32]. Cells, between passage 5-25, were plated at a density of $1 \times 10^{5} / \mathrm{ml}$ for all experiments. Before experimentation, the cells rested between 12 and $24 \mathrm{~h}$ within an incubator set at $37{ }^{\circ} \mathrm{C}$ and a humidified atmosphere augmented with $5 \% \mathrm{CO}_{2}$.

Primary microglia cultures were isolated from 2-day-old Sprague-Dawley rat pups yielding 90\% purity according to methods previously described by our laboratory [33]. All animal protocols received approval from the Uniformed Services University of the Health Sciences (USUHS) Institutional Animal Care and Use Committee (IACUC). Cells were maintained at $37{ }^{\circ} \mathrm{C}$ and a humidified atmosphere augmented with $5 \% \mathrm{CO}_{2}$ in media containing Dulbecco's modified Eagle medium (Gibco), 1\% L-glutamine (Gibco), 1\% sodium pyruvate (Gibco), 10\% FBS (Hyclone), and 1\% penicillin and streptomycin (Thermo Fisher Scientific). Media replacement occurred every 3-5 days. 
Primary cortical neurons were prepared according to protocols previously described [34] with a reported purity of approximately $88 \pm 4 \%$. Briefly, brains were removed from P18 pregnant Sprague-Dawley rat embryos, then cerebellums and meninges were removed prior to trypsinization. Cells were cultured in neurobasal media (Invitrogen, Carlsbad, CA) supplemented with B-27 (Invitrogen), $0.2 \%$ L-glutamic acid monosodium salt hydrate (Sigma), $0.25 \%$ glutamine (Invitrogen), and $1 \%$ antibiotic-antimycotic Solution Cellgro ${ }^{\circ}$ (Corning, Manasas, VA). The cells were plated at a density of $5 \times 10^{5}$ cells $/ \mathrm{ml}$ onto poly-D-lysine (Sigma) treated cell culture plates. All neuronal cultures are fed on day 2 and day 4 with $500 \mu$ of neurobasal media (Invitrogen), 1\% antibiotic-antimycotic solution Cellgro ${ }^{\circ}$ (Corning), and $0.25 \%$ glutamine (Invitrogen). Cells were subjected to 24-h co-culture with microglia on day 7 and then harvested for analysis. PC12 neuron-like cells were cultured in DMEM (Gibco) with $5 \%$ fetal bovine serum (Hyclone), 5\% heat-inactivated horse serum (HIHS, Gibco), and $1 \%$ penicillin with streptomycin (Thermo Fisher Scientific). Cells were passaged every 3-4 days between passage 5 and 20. Differentiation protocols were conducted as previously described [35]. On day 7, differentiated cells were subjected to co-culture with microglia.

Co-culture experimentation between microglia and primary neurons or PC12's was accomplished by plating BV2s into Costar $0.4 \mu \mathrm{m}$ transwells (Corning Incorporated, Corning, NY) at a density of $1 \times 10^{5}$ cells $/ \mathrm{ml}$ and treated as described above. After 24 h, BV2 media containing the experimental treatments was replaced with serum-free media and the transwells were transferred to neuronal cultures possessing only $500 \mu \mathrm{l}$ of media. The BV2's were co-cultured with the neurons for $24 \mathrm{~h}$ and then discarded.

\section{Treatment}

Concentration curves of lipopolysaccharide (LPS), and deferoxamine mesylate (DFO) (Sigma-Aldrich, St. Louis, $\mathrm{MO}$ ), were conducted to determine the optimal concentration for each chemical (data not shown). Experimental groups were treated with $\mathrm{FeSO}_{4}$ tetrahydrate (100 $\mu \mathrm{M}$, Thermo Fisher Scientific), LPS serotype 026:B6 (100 ng/ml, Sigma-Aldrich), ferrous ammonium sulfate $\left(\mathrm{Fe}\left(\mathrm{NH}_{4}\right)_{2}\left(\mathrm{SO}_{4}\right)_{2}\right) \quad(100 \mu \mathrm{M}$, Fisher Scientific), (sodium sulfate) $\mathrm{Na}_{2} \mathrm{SO}_{4}(100 \mu \mathrm{M}$, Fisher Scientific), or DFO (250 $\mu \mathrm{M}$, Sigma-Aldrich). All treatments were added simultaneously and all tests were performed $24 \mathrm{~h}$ after treatment. In subsequent experiments, microglia were treated with GKT137831 (50 $\mu \mathrm{M}$, Genkyotex, Geneva, Switzerland), GSK2795039 (25 $\mu \mathrm{M}$, GlaxoSmithKline, Brentford, UK), or vehicle dimethyl sulfoxide (DMSO) $(\leq 1 \%$, Sigma-Aldrich). These treatments were given simultaneously to the cultures and all assays were conducted $24 \mathrm{~h}$ afterwards.

\section{Reactive oxygen species assays}

The ROS assay $2^{\prime}, 7^{\prime}$-dichlorodihydrofluorescein diacetate $\left(\mathrm{H}_{2} \mathrm{DCFDA}\right.$, Thermo Fisher $)$ was performed at $10 \mu \mathrm{M}$ concentration with minor modifications to the manufacturer's instructions. The cells were incubated for $45 \mathrm{~min}$ with the assay components and fluorescence emission was measured on a FLUOstar (BMG LABTECH Inc., Cary, NC) plate reader.

\section{Cell death assays}

Cell culture media was assessed for lactate dehydrogenase (LDH) $24 \mathrm{~h}$ after treatment using the Cytotox 96 assay (Promega) according to the manufacturer's instructions. Absorbance was measured using a ChroMate microplate reader (Awareness Technology Inc., Palm City, FL).

\section{Cell proliferation count}

Cells were detached with Accutase (Innovative Cell Technologies, San Diego, CA) and counted by a TC 20 (Bio-Rad Laboratories, Hercules, CA) automated cell counter.

\section{Immunocytochemistry}

Cells were fixed with $4 \%$ paraformaldehyde, and rinsed twice with $1 \times$ PBS. Primary antibodies included rabbit CD86 ( $1 \mu \mathrm{l} / \mathrm{ml}$; Abcam, Cambridge, MA), rat CD206 (5 $\mu \mathrm{l} / \mathrm{ml} ;$ Abcam), rabbit Beta III Tublin $(5 \mu \mathrm{l} / \mathrm{ml}$, Abcam), rabbit anti-cleaved caspase-3 $(0.5 \mu \mathrm{l} / \mathrm{ml}$; Abcam), and mouse NeuN (10 $\mu \mathrm{l} / \mathrm{ml}$; Millipore, Billerica, MA). In addition, cell nuclei were stained with DAPI (0.5 $\mathrm{\mu l} / \mathrm{ml}$; Life Technologies, Carlsbad, CA). Fluorescently tagged secondary antibodies (Invitrogen) were visualized with an Olympus BX43 fluorescent microscope with a CellSens Standard imaging program. Five randomly selected images were taken per sample and each image was qualitatively assessed for overall fluorescence. NeuN-positive cells were manually counted using $\mathrm{NIH}$ ImageJ (Bethesda, MD).

\section{Protein analysis}

Cells were rinsed, lysed, and proteins were isolated with cell lysis buffer (Cell Signaling Technology; Danvers, MA) containing protease inhibitors (Thermo Scientific). For Western blotting, proteins were separated by gel electrophoresis with polyacrylamide gels (Bio-Rad) and transferred to nitrocellulose membranes (Bio-Rad). Rabbit DMT1 (15 $\mu \mathrm{l} / \mathrm{ml}$; Abcam) was detected with a secondary antibody conjugated with HRP. Resultant bands were captured with the Simple Protein FluroChem E system and analyzed using densitometry (NIH ImageJ) and normalized to a loading control (0.5 $\mu \mathrm{l} / 5 \mathrm{ml}$, mouse GAPDH, Millipore). 


\section{Intracellular iron detection}

At 6 and $24 \mathrm{~h}$ after incubation with FeSO4, intracellular total iron concentration was assessed using the Iron Assay Kit (Abcam), as per the manufacturer's instructions. Briefly, cells were washed with cold PBS then collected in $700 \mu \mathrm{l}$ assay buffer and incubated on ice for 5 min. Following, samples were centrifuged to remove debris. Resultant supernatant then was processed with the kit for detection of total iron, detected at $600 \mathrm{~nm}$ on a ChroMate microplate reader.

\section{Cytokine and chemokine analysis}

For cytokine analysis, $1000 \mu \mathrm{g}$ of protein per sample was subjected to a Proteome Profiler Mouse Cytokine Array (R\&D Systems, Minneapolis, MN) according to the manufacturer's instructions. Resultant dots were analyzed using densitometry (NIH Image J). All dots were normalized to manufacturer's standardization regions within the membranes.

\section{Fluorescence-activated cell sorting}

Cells were harvested using Accutase (Innovative Cell Technologies) and stained for viability with FVS510 (0.5ul $/ \mathrm{ml}$; BD Biosciences) and the following antibodies conjugated with fluorophores: CD86 with PE $(0.5 \mu \mathrm{l} / \mathrm{ml}$; BD Biosciences), and CD206 with AF647 (1 $\mu \mathrm{l} / \mathrm{ml}$; BD Biosciences). Samples were analyzed with a BD Bioscience fluorescence-activated cell sorting (FACS) cytometer. All data collected were analyzed with FlowJo version 10.1 (FLOWjo, Ashland, OR).

\section{Comparative RT-PCR}

TRIzol $^{\oplus}$ (Invitrogen, Carlsbad, CA) was used to extract mRNA from the cells and mRNA concentrations were measured by a Nanodrop system (Thermo Scientific). A complementary DNA (cDNA) conversion kit (Applied Biosciences, Waltham, MA) used a Veriti thermal cycler (Applied Biosciences) to convert $1 \mu \mathrm{g}$ of mRNA into cDNA, according to the manufacturer's suggested protocol. All quantitative real-time PCR (qRT-PCR) data was procured through StepOnePlus Real-Time PCR System (Applied Biosciences), according to the manufacturer's suggested protocol. All primers were designed by the Primer-Blast tool and synthesized by Integrated DNA Technologies (IDT, Coralville, IA) and listed in Table 1.
CD86 measured by Taqman probe (Thermo Fisher) according to manufacturer's instructions.

\section{Statistics}

All experiments were repeated in triplicate unless otherwise stated. All quantitative data are presented as mean \pm standard error of the mean. Data were subject to a one-way analysis of variance (ANOVA) with Tukey's or Dunnett's multiple comparison's post-hoc test when appropriate, unless otherwise stated. All statistical tests were performed using the GraphPad Prism Program, Version 6.0 h for Macintosh (GraphPad Software, San Diego, CA). A $p$ value $<0.05$ was considered statistically significant.

\section{Results}

Iron independently induces ROS production and accentuates LPS-derived ROS synthesis

Microglia are the primary ROS-producing cells in the $\mathrm{CNS}$ in response to trauma. Given that they possess the necessary machinery to incorporate iron under basal and LPS-stimulated conditions (Additional file 1: Figure S1), we examined the effect of iron on microglial ROS production. Primary rat microglia cultures were exposed to the $\mathrm{Fe}^{2+}$ donor, $\mathrm{FeSO}_{4}$, LPS, or both for $24 \mathrm{~h}$. We detected a significant ROS accentuation among the cells with $\mathrm{FeSO}_{4}$ exposure that was similar to LPS exposure (Ctrl vs. $\mathrm{FeSO}_{4}, p=0.0027$; Ctrl vs. LPS, $p=0.0023$, one-way ANOVA with Tukey's post-hoc test, Fig. 1a). Combining $\mathrm{FeSO}_{4}$, with LPS for $24 \mathrm{~h}$ resulted in a significant elevation of ROS release in comparison to either $\mathrm{FeSO}_{4}$ or LPS alone $\left(\mathrm{FeSO}_{4}\right.$ vs $\mathrm{FeSO}_{4}+\mathrm{LPS}, p<0.0001$; LPS vs $\mathrm{FeSO}_{4}+\mathrm{LPS}, p<0.0001$, one-way ANOVA with Tukey's post-hoc test, Fig. 1a). Further, administration of the iron chelating agent DFO resulted in significant reduction in ROS production in cells that were exposed to $\mathrm{FeSO}_{4}\left(\mathrm{FeSO}_{4}\right.$ vs $\mathrm{FeSO}_{4}+\mathrm{DFO} p=0.0030 ; \mathrm{FeSO}_{4}+\mathrm{LPS}$ vs $\mathrm{FeSO}_{4}+\mathrm{LPS}+\mathrm{DFO} p<0.0001$, one-way ANOVA with Tukey's post-hoc test, Fig. 1a).

To determine if the microglial cell line, BV2, responded similarly, BV2 cells were exposed to 0 (control), 10, 25, 50, or $100 \mu \mathrm{M} \mathrm{FeSO}$, with and without LPS. We found that microglia treated with increasing doses of $\mathrm{FeSO}_{4}$ have increased $\mathrm{ROS}$ production, reaching significance at a dose of $100 \mu \mathrm{M}$. A significant increase in ROS was detected among the groups treated with

Table 1 Selected gene sequences for RT-PCR

\begin{tabular}{llll}
\hline Gene & Symbol & Forward (5'-3') & Reverse $\left(5^{\prime}\right.$-3') \\
\hline Ferritin, light polypeptide 1 & Ftl & CGGAGGGTCAACATGCTATAA & GGAAGCGAGTACAGTGGGAA \\
Ferritin, heavy polypeptide 1 & Fth1 & CAGTGCTTGAACGGAACCC & GTGGTAGTTCTGGCGCACT1 \\
Mannose receptor & Mrc1 (CD206) & GTGGAGTGATGGAACCCCAG & CTGTCCGCCCAGTATCCATC \\
Arginase 1 & Arg1 & GTGAAGAACCCACGGTCTGT & GCACCACACTGACTCTTCCA \\
Chitinase-like 3 & Chil3 (YM1) & ATGGAAGTTGGACCTGCCC & TCCACAGATTCTTCCTCAAAAGC \\
\hline
\end{tabular}




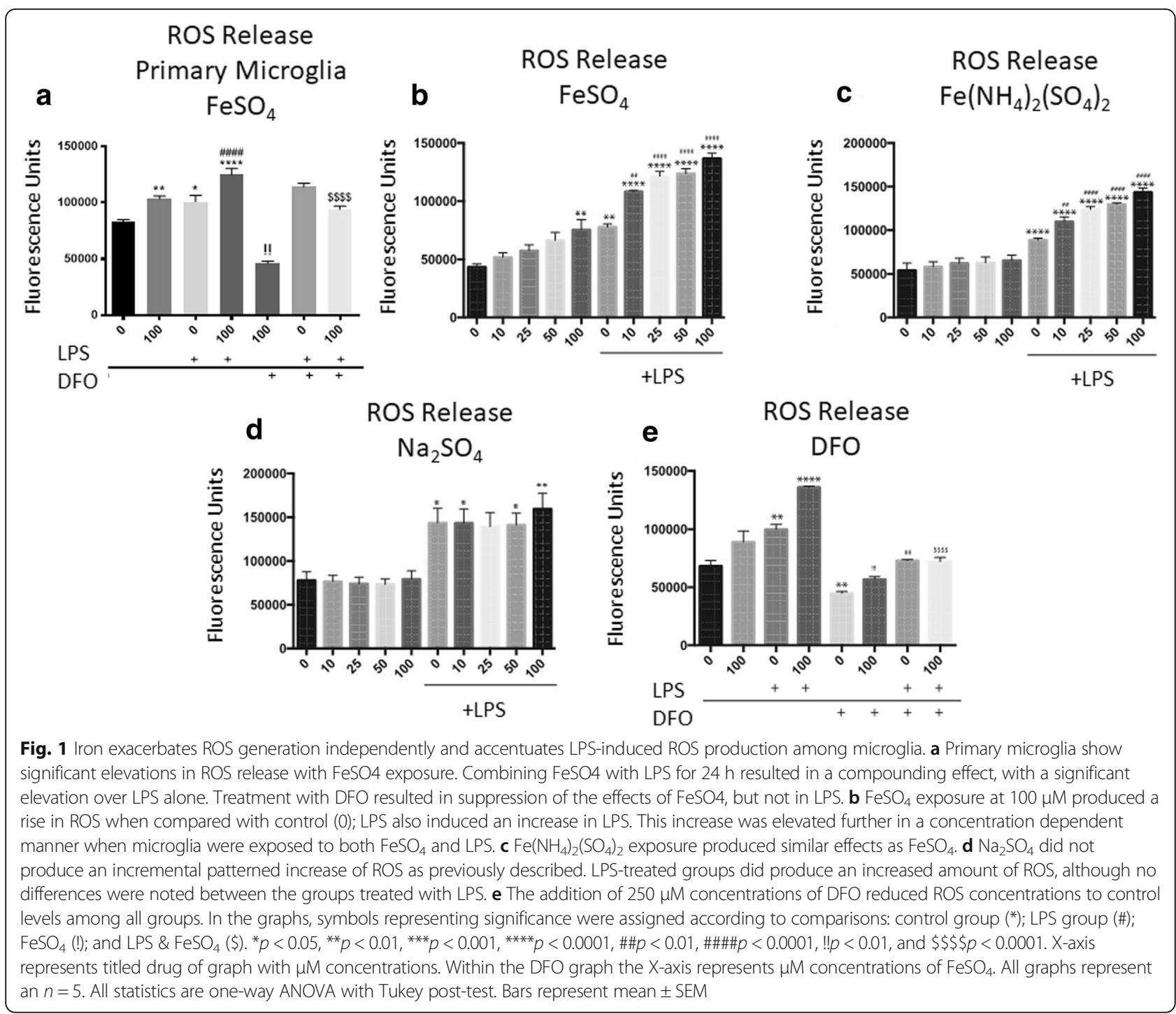

only $100 \mu \mathrm{M} \mathrm{FeSO}_{4}\left(\mathrm{Ctrl}\right.$ vs $100 \mu \mathrm{M} \mathrm{FeSO}_{4}, p=0.0047$; one-way ANOVA with Tukey's post-hoc test, Fig. 1b). LPS induced the production of ROS as expected (Ctrl vs. LPS, $p=0.0023$, Fig. 1 b); $\mathrm{FeSO}_{4}$ addition to LPS led to an incremental elevation above the LPS-induced ROS in a concentration-dependent fashion (LPS vs: LPS \& $10 \mu \mathrm{M} \mathrm{FeSO}, p=0.0067$; LPS \& $25 \mu \mathrm{M}$ $\mathrm{FeSO}_{4}, p<0.0001$; LPS \& $50 \mu \mathrm{M} \mathrm{FeSO}_{4}, p<0.0001$; LPS \& $100 \mu \mathrm{M} \mathrm{FeSO}, p<0.0001$; one-way ANOVA with Tukey's post-hoc test, Fig. 1b). As these initial experiments showed similar results with BV2 cells, we continued experiments utilizing this cell line.

To ensure this phenomenon was not unique to $\mathrm{FeSO}_{4}$, another $\mathrm{Fe}^{2+}$ donor, ferrous ammonium sulfate $\left(\mathrm{Fe}\left(\mathrm{NH}_{4}\right)_{2}\left(\mathrm{SO}_{4}\right)_{2}\right)$, was tested. A similar pattern of increase in ROS within the groups treated with both LPS and $\mathrm{Fe}\left(\mathrm{NH}_{4}\right)_{2}\left(\mathrm{SO}_{4}\right)_{2}$ was observed (Ctrl vs. LPS, $p<0.0001$; LPS vs: LPS \& $10 \mu \mathrm{M} \mathrm{FeSO}, p=0.0033$;
LPS \& $25 \mu \mathrm{M} \quad \mathrm{FeSO}_{4}, p<0.0001 ;$ LPS \& $50 \mu \mathrm{M}$ $\mathrm{FeSO}_{4}, p<0.0001$; LPS \& $100 \mu \mathrm{M} \mathrm{FeSO}, p<0.0001$; one-way ANOVA with Tukey's post-hoc test, Fig. 1c).

Next, to ensure that results were a result of the iron inclusion, a control experiment using inert sodium attached to the sulfate carrier of both iron donors was evaluated by exposing cultures to $\mathrm{Na}_{2} \mathrm{SO}_{4}$. While most groups with LPS treatment demonstrated significant increases in ROS production (Ctrl vs. LPS, $p=0.0342$; Ctrl vs. LPS \& $10 \mu \mathrm{M} \mathrm{Na} \mathrm{SO}_{4}, p=0.0359$; Ctrl vs. LPS \& $50 \mu \mathrm{M} \mathrm{Na} \mathrm{SO}_{4}, p<0.046$; Ctrl vs. LPS \& $100 \mu \mathrm{M}$ $\mathrm{Na}_{2} \mathrm{SO}_{4}, \quad p<0.0052$, one-way ANOVA with Tukey's post-hoc test, Fig. 1d), there were no differences in $\mathrm{Na}_{2} \mathrm{SO}_{4}$ treated cells.

Finally, to confirm that these effects were the effect of iron, DFO $(250 \mu \mathrm{M})$, an iron chelator, was assessed. The addition of DFO ameliorated the ROS accentuation by reducing ROS levels among all groups at or below 
control levels (Ctrl vs. DFO, $p=0.0065 ; \mathrm{FeSO}_{4}$ vs. DFO $\& \mathrm{FeSO}_{4}, p=0.0007$; LPS vs. DFO \& LPS, $p=0.0026$; $\mathrm{FeSO}_{4}$ \& LPS vs. DFO \& $\mathrm{FeSO}_{4}$ \& LPS, $p<0.0001$; one-way ANOVA with Tukey's post-hoc test, Fig. 1e).

ROS production can be reduced if the numbers of cells are different between experimental conditions. To confirm that iron and/or LPS treatments were not resulting in cell death and subsequent reduction of ROS production, we performed an LDH assay and found no differences in LDH between groups (Additional file 2: Figure $\mathrm{S} 2$ ), indicating that cell death is not impacting ROS production. Increased number of cells can also result in increased ROS production upon stimulation. To verify that the increase in ROS was not due to cell number following stimulation, we counted cells in each group. No significant differences were detected between the groups (data not shown).

\section{Iron does not induce polarization alterations in LPS- stimulated cells}

Microglia rapidly respond to alterations of environmental homeostasis by transitioning to a pro-inflammatory state. This inflammatory state is accompanied by increased cytokine production and has been shown to promote neuronal death and exacerbate the injury. To determine how iron alters microglial polarization in response to LPS, we examined gene expression of several M1 (CD86) and M2 (Arg1, YM1, and CD206) markers $24 \mathrm{~h}$ after treatment. LPS resulted in increased gene expression of CD86 and an overall reduction of the anti-inflammatory markers: CD86 (Ctrl vs. LPS, $p=0.0402$, Fig. 2a), ARG1 (Ctrl vs. LPS, $p=0.0002$, Fig. 2b), YM1 (Ctrl vs. LPS, $p<0.0001$, Fig. 2c), and CD206 (Ctrl vs. LPS, $p=0.0009$, Fig. 2d) (All statistics are two-way ANOVA with Tukey's post-test). Iron alone did not significantly influence gene expression within any group. In addition, comparisons between LPS versus LPS with iron groups yielded no significant differences, although a slight increase in CD86 was observed with the combination of LPS and iron.

Next, we determined polarization profiles by flow cytometry. CD86 expression in cells treated with LPS alone was slightly elevated over control, although this did not reach statistical significance (Ctrl vs. LPS, $p=0.0611$, two-way ANOVA with Tukey's post-hoc test, Fig. 2e). We found the intensity of CD86 staining among groups treated with a combination of LPS and iron increased significantly (Ctrl vs. $\mathrm{FeSO}_{4} \& \mathrm{LPS}, p=0.0331$, two-way ANOVA with Tukey's post-hoc test, Fig. 2e). We detected a significant reduction in CD206 fluorescence staining among the groups (Ctrl vs. LPS, $p<0.0009$; Ctrl vs. $\mathrm{FeSO}_{4} \&$ LPS, $p<0.0047$, two-way ANOVA with Tukey's post-hoc test, Fig. 2f), although no significant effect of iron was found.
To further investigate M1 and M2 marker expression, immunocytochemistry was performed $24 \mathrm{~h}$ after incubation with LPS and/or $\mathrm{FeSO}_{4}$. Qualitative analysis of the immunocytochemistry revealed a greater expression of CD86 within LPS groups when compared with controls (Fig. 2g). Analysis of CD206 suggested a reduction in its expression when cultures were exposed to LPS. When combined, $\mathrm{FeSO}_{4}$ and LPS did not appear to intensify the CD86 fluorescence binding nor did it further reduce CD206 expression.

To confirm these results in primary cells, immunocytochemistry for CD86 was performed. While LPS stimulation did lead to a qualitative increase in CD86 protein expression, addition of $\mathrm{FeSO}_{4}$ to LPS-treated primary microglia did not induce a further increase in CD86 (Additional file 3: Figure S3). In fact, qualitative evaluation showed a slight decrease in $\mathrm{FeSO}_{4}$-treated cells. No marked difference in cell number was noted in any treatment condition, as assessed by DAPI nuclear staining.

Pro-inflammatory microglia produce cytokines that can be detrimental to neuronal survival. We examined the role of iron on cytokine production in the absence and presence of iron and/or LPS. Six cytokines associated with the pro-inflammatory response were increased by LPS (Table 2). Overall, LPS had a pro-inflammatory effect on microglia-inducing synthesis of IL-1 $\alpha$ (Ctrl vs. LPS, $p=0.0055)$, IL- $1 \beta$ (Ctrl vs. LPS, $p=0.006$ ), IP-10 (Ctrl vs. LPS, $p=0.0053$ ), RANTES (Ctrl vs. LPS, $p=0.0006)$, and TNF $\alpha$ (Ctrl vs. LPS, $p=0.0017$, two-way ANOVA with Tukey's post-hoc test, Fig. 2h). Iron alone did not have an effect on any of the cytokines tested, further supporting the idea that iron's effects are primarily related to ROS release.

\section{Iron increases neurotoxicity among co-cultures}

Given that activated microglia promote oxidative stress-induced cell death in neurons, we wanted to evaluate the role of iron on microglia-induced neuronal cell death. We found that when LPS-treated microglia were indirectly co-cultured with primary neuronal cells via transwell plates, the number of $\mathrm{NeuN}^{+}$cells were decreased. Immunocytochemistry for NeuN-positive cells revealed that the addition of iron to the LPS stimulated microglia further reduced NeuN staining, with an almost complete loss of NeuN staining (Fig. 3; no microglia (NM) vs. $\mathrm{FeSO}_{4}$ \& LPS, $p=0.0035$; Ctrl vs. FeSO4 \& LPS, $p=0.0027$ ). This effect was reversed by the administration of the iron chelator DFO to the stimulated microglia, prior to incubation with neurons (LPS \& $\mathrm{FeSO}_{4}$ vs. DFO \& LPS \& $\mathrm{FeSO}_{4}, p=0.0064$, Fig. 3).

Beta III tubulin is a protein found in neurons that allow visualization of the neurite networks. Staining of primary neurons following co-culture with microglia treated with iron resulted in a marked loss of Beta III 


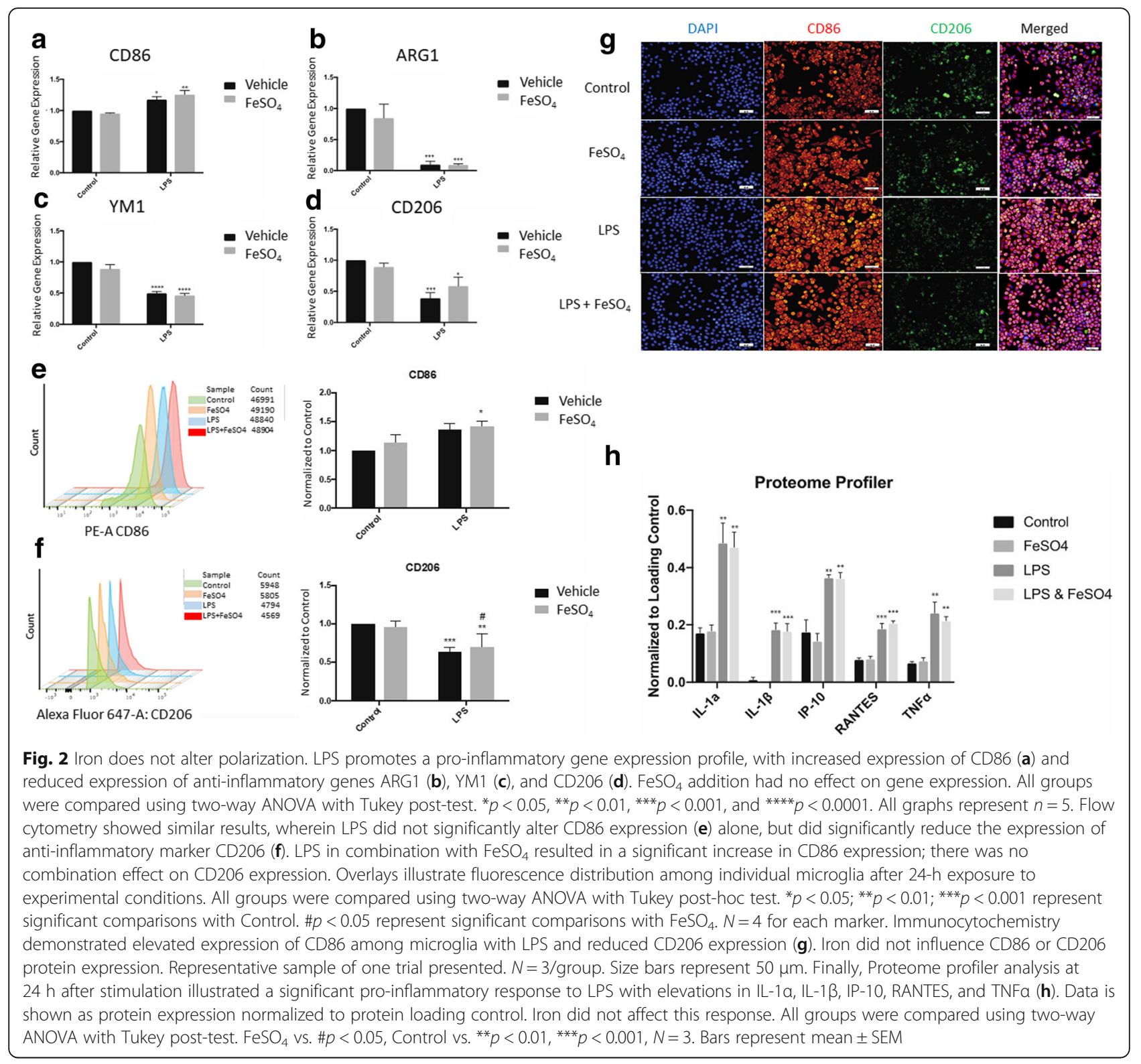

tubulin staining. LPS incubation with microglia prior to co-culture further reduced staining, and staining was almost completely lost with the combination of iron and LPS. This observation was again completely reversed with administration of DFO to microglia (Fig. 3a).

NOX2 or NOX4 inhibition reduces ROS production among microglia exposed to $\mathrm{FeSO}_{4}$ and LPS

NOX2 is an inducible superoxide generating enzyme that produces hydroxyl radicals and ROS; its structure suggests a partial dependence on iron [36]. Given that NOX2 is upregulated following LPS treatment and iron can accentuate LPS-induced ROS production (Additional file 1: Figure S1a), we treated primary microglia with a NOX2 inhibitor, GSK279503, and measured ROS production.
$\mathrm{FeSO}_{4}$ and LPS resulted in a significant increase in the presence of $\operatorname{ROS}(p=0.0002$, one-way ANOVA with Sidak's multiple comparisons post-hoc test, Fig. 4a). NOX2 inhibition of LPS/iron-treated microglia resulted in ROS levels that returned to those of the control group $\left(\mathrm{FeSO}_{4}\right.$ \& LPS vs. $\mathrm{FeSO}_{4}$ \& LPS \& GSK, $p=0.0015$, one-way ANOVA with Sidak's multiple comparisons post-hoc test, Fig. 4a).

In BV2 cells, $\mathrm{FeSO}_{4}$ and/or LPS also resulted in a significant increase in the presence of ROS (DMSO vs: FeSO4, $p=0.0089$; FeSO4 \& LPS, $p<0.0001$, one-way ANOVA with Tukey's post-hoc test, Fig. 4b). NOX2 inhibition of LPS/iron-treated microglia resulted in ROS levels that returned to those of the control group $\left(\mathrm{FeSO}_{4}\right.$ \& LPS vs. $\mathrm{FeSO}_{4}$ \& LPS \& GSK, $p<0.0001$, one-way 
Table 2 Cytokine and chemokine responses

\begin{tabular}{|c|c|c|c|c|}
\hline Protein & Control Mean \pm SEM & $\mathrm{FeSO}_{4}$ Mean \pm SEM & LPS Mean \pm SEM & $\mathrm{LPS} \& \mathrm{FeSO}_{4}$ Mean $\pm \mathrm{SEM}$ \\
\hline G-CSF & ND & ND & $0.074 \pm 0.013$ & $0.067 \pm 0.008$ \\
\hline SICAM-1 & $0.690 \pm 0.254$ & $0.813 \pm 0.32$ & $0.813 \pm 0.324$ & $0.698 \pm 0.26$ \\
\hline IL-1a & $0.171 \pm 0.018$ & $0.179 \pm 0.021$ & $0.486 \pm 0.069$ & $0.47 \pm 0.053$ \\
\hline $\mid L-1 \beta$ & ND & ND & $0.183 \pm 0.023$ & $0.177 \pm 0.027$ \\
\hline IL-1ra & $0.934 \pm 0.052$ & $0.87 \pm 0.059$ & $1.165 \pm 0.023$ & $1.18 \pm 0.104$ \\
\hline IP-10 & $0.175 \pm 0.042$ & $0.143 \pm 0.026$ & $0.365 \pm 0.011$ & $0.362 \pm 0.021$ \\
\hline M-CSF & $0.097 \pm 0.01$ & $0.107 \pm 0.005$ & $0.085 \pm 0.004$ & $0.083 \pm 0.011$ \\
\hline JE & $0.573 \pm 0.05$ & $0.535 \pm 0.055$ & $0.619 \pm 0.049$ & $0.568 \pm 0.062$ \\
\hline MIP-1a & $0.941 \pm 0.016$ & $1.07 \pm 0.115$ & $0.849 \pm 0.064$ & $0.769 \pm 0.019$ \\
\hline MIP-1 $\beta$ & $0.023 \pm 0.011$ & $0.021 \pm 0.012$ & $0.113 \pm 0.028$ & $0.106 \pm 0.024$ \\
\hline MIP-2 & ND & ND & $0.072 \pm 0.01$ & $0.072 \pm 0.002$ \\
\hline RANTES & $0.079 \pm 0.005$ & $0.081 \pm 0.009$ & $0.186 \pm 0.019$ & $0.206 \pm 0.008$ \\
\hline SDF-1 & $0.039 \pm 0.008$ & $0.038 \pm 0.007$ & $0.04 \pm 0.009$ & $0.036 \pm 0.006$ \\
\hline TIMP-1 & $0.052 \pm 0.0007$ & $0.057 \pm 0.01$ & $0.058 \pm 0.0029$ & $0.063 \pm 0.003$ \\
\hline TNFa & $0.067 \pm 0.005$ & $0.074 \pm 0.011$ & $0.241 \pm 0.038$ & $0.214 \pm 0.014$ \\
\hline TREM-1 & $0.08 \pm 0.002$ & $0.08 \pm 0.007$ & $0.103 \pm 0.003$ & $0.091 \pm 0.008$ \\
\hline
\end{tabular}

Values represent relative expression of cytokines and chemokines normalized to manufacturer's controls after stimulation. No significant differences were noted between control and $\mathrm{FeSO}_{4}$ or between LPS and FeSO4 \& LPS groups. All groups were compared using two-way ANOVA with Tukey's post-test. All groups represent $N=3$. All data presented as mean $\pm \mathrm{SEM}$

ANOVA with Tukey's post-hoc test, Fig. 4b). No significant differences were noted between groups by LDH assay or cell counts among cultures (Fig. 4b).

NOX4, unlike NOX2, is constitutively expressed and catalyzes the formation of hydrogen peroxide [36]. Given that hydrogen peroxide can oxidize iron to its reactive state, we sought to determine if NOX4 inhibition could block iron-accentuated ROS production in microglia. We found that NOX4 inhibition with GKT137831 significantly reduced the iron-induced ROS production in primary cells $\left(\mathrm{FeSO}_{4}\right.$ \& LPS vs. $\mathrm{FeSO}_{4}$ \& LPS \& GSK, $p=0.0003$, one-way ANOVA with Sidak's multiple comparisons post-hoc test, Fig. 4a).

In BV2 cells, we found a similar result. $\mathrm{FeSO}_{4}$ and/or LPS led to a significant increase in ROS (DMSO vs: $\mathrm{FeSO}_{4}, p<0.0001$; LPS, $p=0.001 ; \mathrm{FeSO}_{4}$ \& LPS, $p<0.0001$, one-way ANOVA with Tukey's post-hoc test, Fig. 4c). Interestingly, GKT137831 treatment alone resulted in a significant reduction in ROS in comparison to the vehicle-treated group (DMSO vs. GKT, $p=0.0055$, one-way ANOVA with Tukey's post-hoc test, Fig. 4c), likely because NOX4 is endogenously active in microglia. In addition, GKT137831 significantly reduced ROS production among microglia simultaneously treated with $\mathrm{FeSO}_{4}$ and LPS $\left(\mathrm{FeSO}_{4}\right.$ \& LPS \& DMSO vs. $\mathrm{FeSO}_{4}$ \& LPS \& GKT, $p<0.0001$, one-way ANOVA with Tukey's post-hoc test, Fig. 4c). No significant differences were noted between groups by LDH assay or cell counts among cultures within the GKT137831 set of experiments (Fig. 4c). While slight differences in ROS responses were noted between experiments represented in Fig. 4b, 4c, likely due to slight variability in the control group as a result of passage number, overall trends were similar between the two studies.

Iron accentuated, microglial-mediated neuronal toxicity is reversed by NOX2 inhibition

To determine the effectiveness of specific NOX isoform inhibition in microglia on neuronal survival, we indirectly co-cultured microglia in transwell plates with primary rat neuron cultures. Microglia pretreated with $\mathrm{FeSO}_{4}$ \& LPS prior to co-culture with neurons resulted in a reduced nuclear staining of DAPI and neuron specific NeuN (Fig. 5a). DAPI-positive cells were quantified and a significant reduction of cells was noted among neuronal cultures co-cultured with microglia treated with $\mathrm{FeSO}_{4}$ \& LPS (DMSO vs. $\mathrm{FeSO}_{4}$ \& LPS \& DMSO, $p=0.0007$; one-way ANOVA with Tukey's post-hoc test, Fig. 5b). NOX2 inhibition by GSK2795039 within microglia prior to co-culture significantly increased the presence of cells within the neuronal cultures $\left(\mathrm{FeSO}_{4}\right.$ \& LPS \& DMSO vs. GSK2795039 \& $\mathrm{FeSO}_{4} \&$ LPS, $p=0.0012$, one-way ANOVA with Tukey's post-hoc test, Fig. 5b). To determine the toxicity specific to neurons, all NeuN-positive cells were counted among the cultures. Significant reductions of primary neuronal cell counts were detected among cultures treated with a 


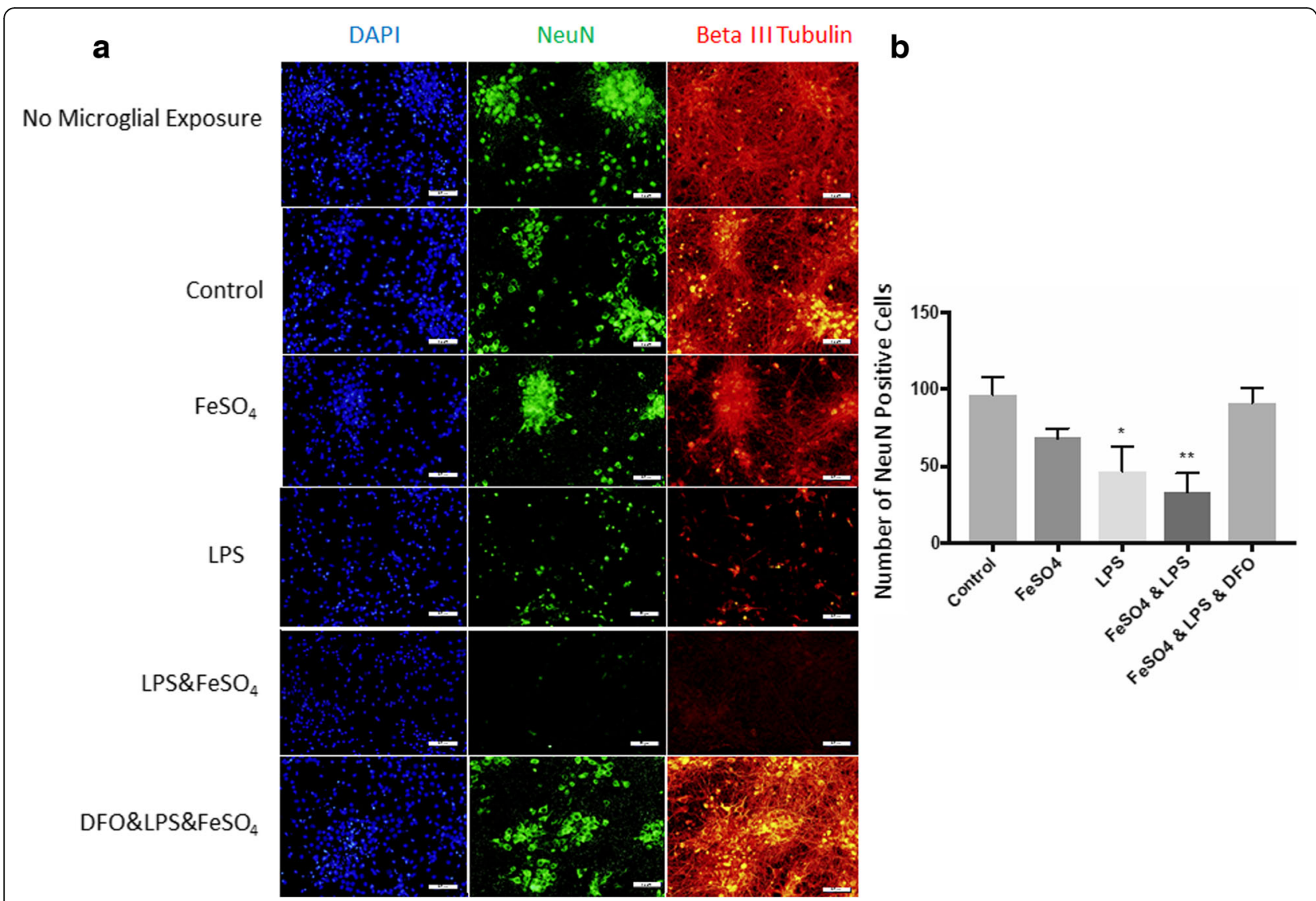

Fig. 3 Microglia treated with LPS \& $\mathrm{FeSO}_{4}$ increased primary neuronal toxicity in co-culture. a Immunocytochemistry of NeuN-positive primary neurons after 24-h microglial co-culture revealed reduced number of neurons and neurite network when exposed to LPS or LPS \& FeSO Although $\mathrm{FeSO}_{4}$ alone did not result in a significant reduction, a lower number of neurons and decreased density of neurite network was noted. The addition of DFO ameliorated the toxic effect of microglia treated with LPS alone and LPS \& FeSO 4 . $\mathbf{b}$ Quantification of NeuN-positive cells illustrated the toxic effect of LPS alone and in combination with $\mathrm{FeSO}_{4}$. Representative sample of 1 trial of 4 shown. Graph represents a sample size of $4 .{ }^{*} p<0.05 ;{ }^{* *} p<0.01$, one-way ANOVA with Tukey's post-hoc test. Bars represent mean \pm SEM. Size bars represent $50 \mu \mathrm{m}$

combination of $\mathrm{FeSO}_{4}$ \& LPS (DMSO vs. $\mathrm{FeSO}_{4}$ \& LPS \& DMSO, $p=0.0027$, one-way ANOVA with Tukey's post-hoc test, Fig. 5c). Interestingly, the addition of GSK2795039 significantly increased the presence of neurons among those treated with $\mathrm{FeSO}_{4}$ \& LPS $\left(\mathrm{FeSO}_{4}\right.$ \& LPS \& DMSO vs. GSK2795039 \& $\mathrm{FeSO}_{4}$ \& LPS, $p<0.0001$, one-way ANOVA with Tukey's post-hoc test, Fig. $5 \mathrm{c}$ ).

Due to the heterogeneous cellular composition of primary neuronal cultures, we utilized robust differentiated PC12 neuronal-like cells in co-culture with microglia to evaluate the severity of cellular toxicity among purified neuronal cell cultures. To evaluate changes in apoptosis following treatment, we analyzed levels of cleaved caspase-3, a known marker of apoptosis. After co-culture for $24 \mathrm{~h}, \mathrm{PC} 12 \mathrm{~s}$ did not display significantly increased levels of cleaved caspase-3-positive cells when co-cultured with microglia previously treated with vehicle, $\mathrm{FeSO}_{4}$, LPS, or GSK2795039 alone. However, a combination $\mathrm{FeSO}_{4}$ \& LPS treatment of microglia prior to co-culture increased the number of cleaved caspase-3-positive cells within the PC12 cultures; the addition of GSK2795039 reversed this effect (Fig. 5d, e). Caspase- 3 gets cleaved and translocated to the nucleus after the induction of apoptosis, so to quantify the differences in apoptosis between treatment groups, all co-localized DAPI and caspase-3-positive cells were counted and the combinatory effect of $\mathrm{FeSO}_{4}$ and LPS resulted in significantly increased levels of apoptosis (DMSO vs FeSO4 \& LPS \& DMSO, $p<0.0001$, one-way ANOVA with Tukey's post-hoc test, Fig. 5e). The addition of the NOX2 inhibitor GSK2795039 significantly reduced the presence of caspase-3-positive PC12 cells (FeSO4 \& LPS \& DMSO vs. GSK2795039 \& FeSO4 $\&$ LPS, $p<0.0001$, one-way ANOVA with Tukey's post-hoc test, Fig. 5e). 


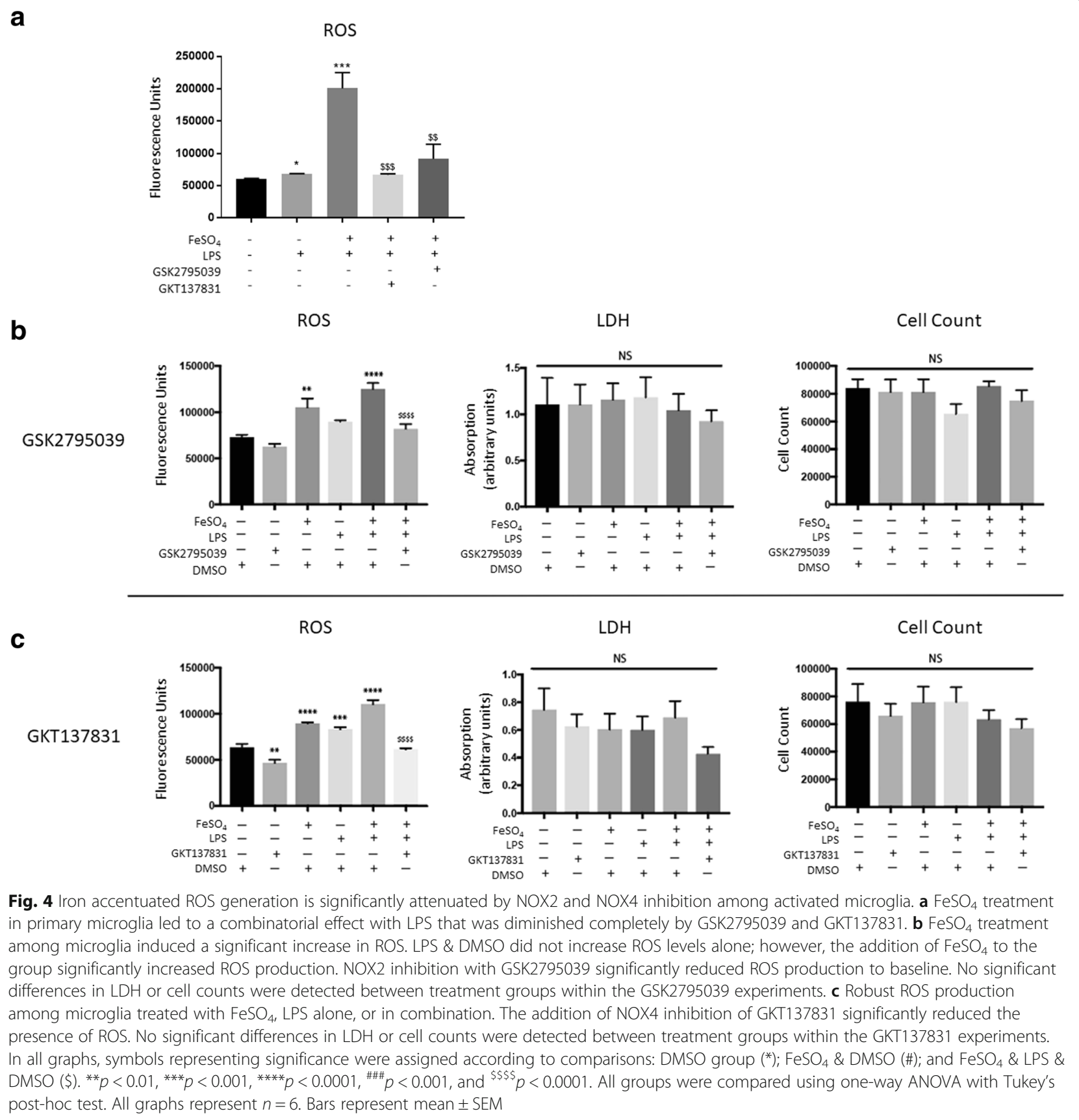

Iron accentuated, microglial-mediated neuronal toxicity is reversed by NOX4 inhibition

Next, we evaluated the contribution of NOX4 to microglial-mediated neurotoxicity by inhibiting NOX4 with GKT137831. Primary neuronal cultures displayed fewer DAPI-positive and NeuN-positive nuclei after co-culture with microglia treated with LPS or $\mathrm{FeSO}_{4}$ \& LPS. This toxic phenomenon was completely reversed by the addition of GKT137831 (Fig. 6a). The DAPI-positive cells were again counted and a significant reduction in cells were noted among neuron cultures exposed to microglia treated with a combination of $\mathrm{FeSO}_{4}$ \& LPS (DMSO vs. $\mathrm{FeSO}_{4}$ \& LPS \& DMSO, $p=0.0005$, one-way ANOVA with Tukey's post-hoc test, Fig. 6b). While cultures were treated identically as in the NOX2 inhibition experiment, slight variation in cell responses resulted in a lack of significant reduction in DAPI-positive and NeuN-positive cells with LPS treatment alone. However, a similar trend was observed. The addition of GKT137831 resulted in a significant increase in the presence of cells $\left(\mathrm{FeSO}_{4}\right.$ \& LPS \& DMSO vs. GKT137831 \& 


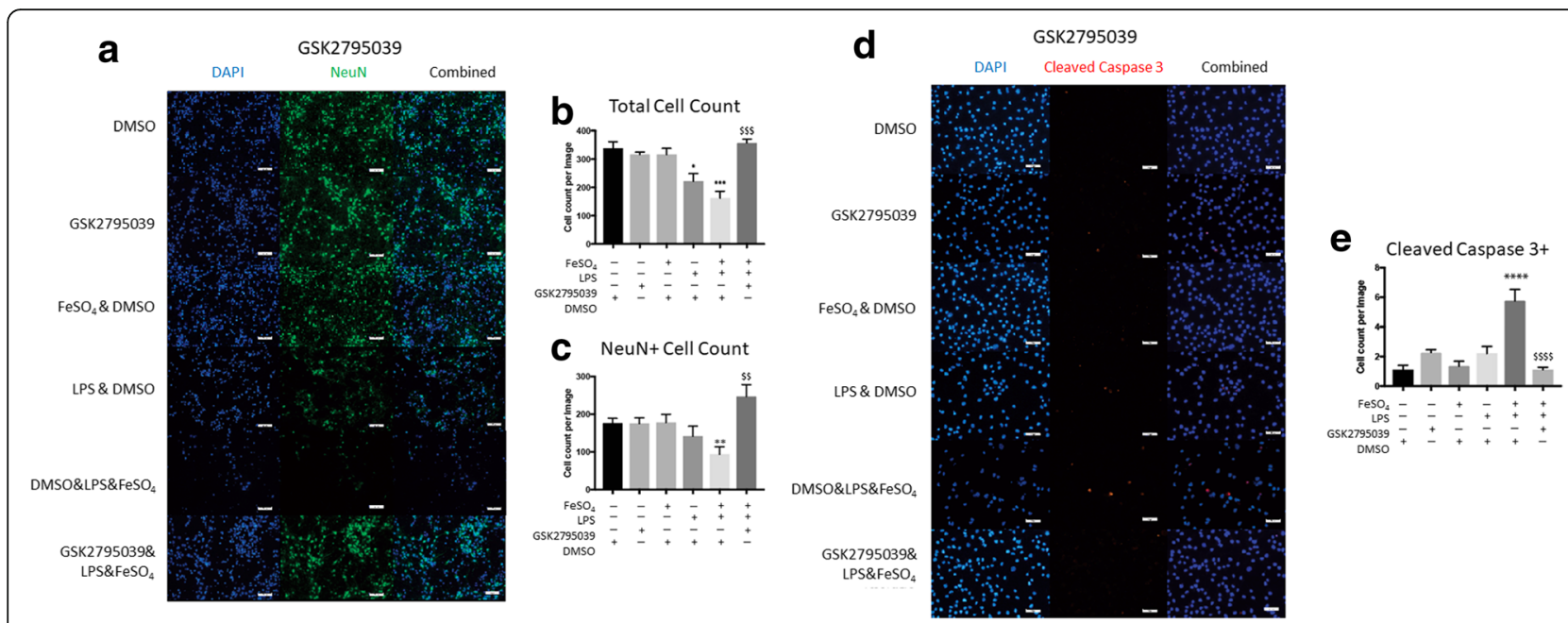

Fig. 5 GSK2795039 improves neuronal survival in co-culture with activated microglia. a Primary neurons were co-cultured with BV2 microglia exposed to FeSO4, DMSO, LPS, and/or GSK2795039. Immunocytochemistry was completed for neurons (NeuN) at $24 \mathrm{~h}$ after co-culture. All groups were counter-stained with DAPI. Qualitatively, a reduction in the expression of NeuN was noted on those groups co-cultured with microglia treated with FeSO4, LPS, or LPS\&FeSO4. The addition of GSK2795039 significantly improved neuronal survival. $\mathbf{b}$ Improved survival among all cell types present in the neuronal culture (DAPI-positive) was observed as increased cell counts in groups treated with GSK2795039. c Quantitative analysis of NeuN-positive cell counts revealed a significant reduction in neuronal cell count after co-culture with microglia treated with $\mathrm{FeSO}_{4}$ \& LPS \& DMSO. GSK2795039 prevented neuronal loss after co-culture and significantly improved neuronal cell counts. d PC12 cells were differentiated to a neuronal like phenotype for one week prior to microglia co-culture. Immunocytochemistry revealed increased staining for cleaved caspase-3 among PC12 co-cultured with BV2 microglia treated with FeSO 4 , LPS, or LPS\&FeSO4. The addition of GSK2795039 to the microglia treatment groups reduced the presence of cells positive for cleaved caspase-3. e Quantitative analysis of cleaved caspase-3 positive $\mathrm{PC1} 2$ cells showed significant increase when co-cultured with microglia treated with $\mathrm{FeSO}_{4}$ \& LPS \& DMSO. However, this increased apoptosis among PC12 cells was reduced by the addition of GSK2795039. All immunocytochemistry images are representative of one trial. All groups were compared using one-way ANOVA with Tukey's post-hoc test. In all graphs, symbols representing significance were assigned according to comparisons: DMSO group $\left({ }^{*}\right)$ and FeSO 4 \& LPS \& DMSO (\$). ${ }^{*} p<0.05,{ }^{* *} p<0.01,{ }^{* * *} p<0.001,{ }^{* * *} p<0.0001,{ }^{\$ \$} p<0.01,{ }^{\$ \$ \$} p<0.001,{ }^{\$ \$ \$ \$} p<0.0001$. All graphs represent $n=4$. Bars represent mean \pm SEM. Size bars represent $50 \mu \mathrm{m}$

$\mathrm{FeSO}_{4}$ \& LPS, $p=0.0002$, one-way ANOVA with Tukey's post-hoc test, Fig. 6b).

NeuN-positive primary neurons were also counted to determine cellular toxicity among neurons. Those neuronal cultures subjected to co-culture with microglia pretreated with $\mathrm{FeSO}_{4}$ \& LPS showed a significant reduction in neuronal cell counts which was ameliorated by the addition of GKT137831 (DMSO vs. $\mathrm{FeSO}_{4} \&$ LPS \& DMSO, $p=0.048 ; \mathrm{FeSO}_{4}$ \& LPS \& DMSO vs. GKT137831 \& $\mathrm{FeSO}_{4} \quad \&$ LPS, $p=0.0438$, one-way ANOVA with Tukey's post-hoc test, Fig. 6c).

PC12 cultures were then subjected to NOX4 inhibition by GKT137831. PC12s qualitatively did not display increased levels of caspase-3-positive cells and were not affected by co-cultured microglia previously treated with vehicle, $\mathrm{FeSO}_{4}$, or GKT137831 alone. LPS and $\mathrm{FeSO}_{4}$ \& LPS groups did present with higher numbers of caspase-3-positive cells, which was reversed with the addition of GKT137831 (Fig. 6d). Quantitative analysis demonstrated that LPS treated microglia caused a significant increase in caspase-3-positive PC12 cells (DMSO vs. LPS \& DMSO, $p=0.0081$, one-way ANOVA with Tukey's post-hoc test, Fig. 6e). This toxicity worsened with the combination $\mathrm{FeSO}_{4}$ and LPS treatment groups (DMSO vs. FeSO4 \& LPS \& DMSO, $p<0.0001$, one-way ANOVA with Tukey's post-hoc test, Fig. 6e) and was reversed with the addition of GKT137831 (FeSO4 \& LPS \& DMSO vs. GKT137831 \& FeSO4 \& LPS, $p<0.0001$, one-way ANOVA with Tukey's post-hoc test, Fig. 6e). In comparison to the NOX2 inhibition experiment (Fig. 5), experimental variability did result in a greater number of cleaved caspase-3-positive PC12 cells in the current experiment, but trends of responses remain the same. This difference may be due to the slight difference in PC12 passage number between NOX2 and NOX4 experimentation.

\section{Discussion}

These data demonstrate that iron contributes to microglial ROS production in a NOX2- and NOX4-dependent manner and that this ROS production, without an accompanying alteration in pro-inflammatory/M1 polarization markers or cytokines, contributes to microglial-induced neurotoxicity. Further, while iron both induces ROS production from microglia and accentuates ROS synthesis from activated microglia, iron did not alter polarization in non-stimulated or LPS stimulated primary microglia or BV2 cells. Importantly, 


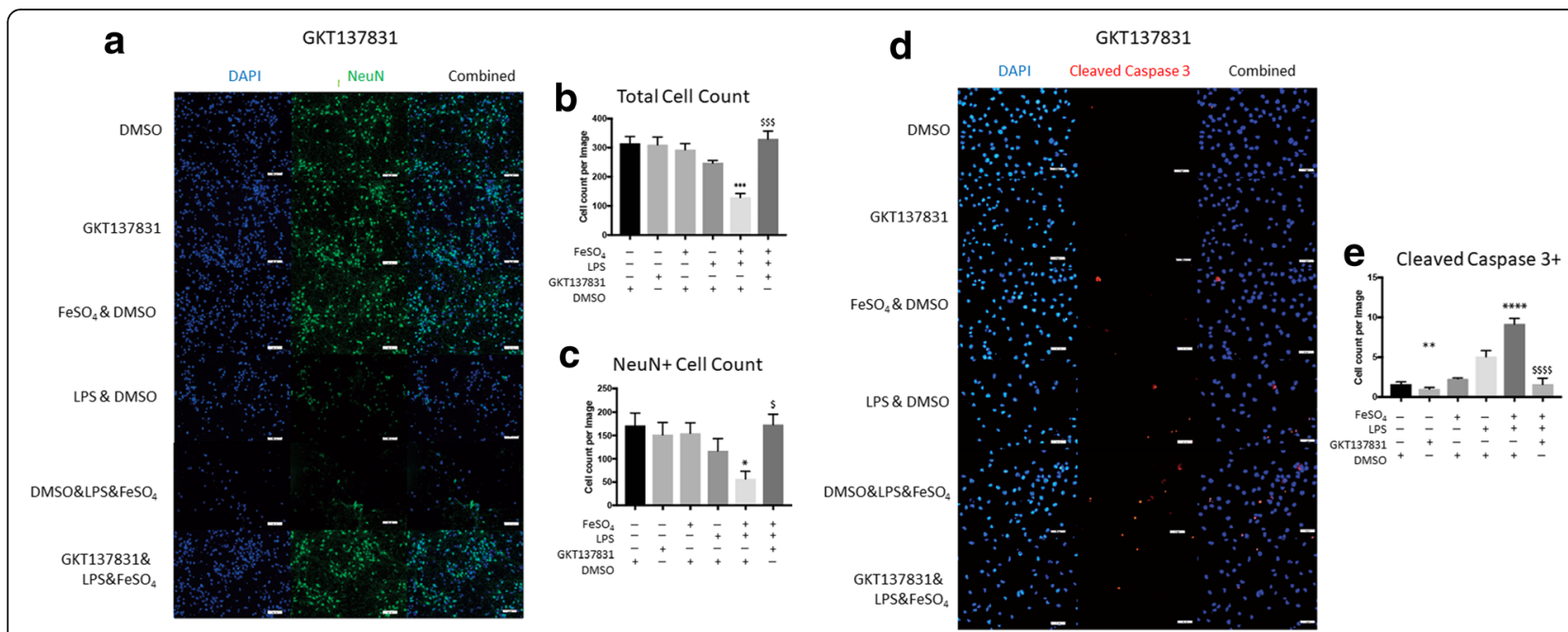

Fig. 6 GKT137831 improves neuronal survivability in co-culture with activated microglia. a Primary neurons were co-cultured with microglia exposed to FeSO4, DMSO, LPS, GKT, or in combination. Immunostaining of neurons after $24 \mathrm{~h}$ co-culture showed a reduction in the expression of NeuN in groups co-cultured with microglia treated with $\mathrm{FeSO}_{4} \&$ LPS \& DMSO. b Improved survivability among all cell types present in the neuronal culture were observed by the increased cell counts in groups treated with GKT137831. c Quantitative analysis of NeuN counts revealed a significant reduction in the presence of neurons after co-culture with microglia treated with $\mathrm{FeSO}_{4}$ \& LPS \& DMSO. GKT137831 reduced neuronal toxicity and prevented neuronal loss after co-culture. $\mathbf{d}$ PC12 cells were differentiated to a neuronal like phenotype for one week prior to microglia co-culture. Immunocytochemistry revealed increased staining among PC12 co-cultured with microglia treated with LPS, or in combination with $\mathrm{FeSO}_{4}$. The addition of GKT137831 to the microglia treatment groups reduced the presence of cells positive for cleaved caspase-3. e Quantitative analysis of PC12 cells positive with cleaved caspase-3 were significantly increased when co-cultured with microglia treated with $\mathrm{FeSO}_{4}$ \& LPS \& DMSO. However, this increased apoptosis among PC12 cells was reversed by the addition of GKT137831. All immunostaining images are representative of one trial. All groups were compared using one-way ANOVA with Tukey post-test. In all graphs, symbols representing significance were assigned according to comparisons: DMSO group (*); $\mathrm{FeSO}_{4}$ \& DMSO (\#); LPS \& DMSO (!); and FeSO 4 \&

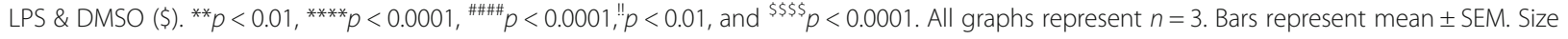
bars represent $50 \mu \mathrm{m}$

despite this lack of polarization change, the data from the current work demonstrate that the elevated ROS leads to increased neuronal death in a BV2-primary neuron co-culture model and can be reversed with iron chelation. NOX2 and NOX4 may be important within the mechanism of iron accentuated ROS production and their inhibition seems to be effective in reducing neuronal damage.

Iron overload prior to CNS injury exacerbates lesion volume and reduces functional recovery, and suggests a potential priming effect by iron [10]. Our data is consistent with these observations in that the presence of iron exacerbated the ROS production among microglia and exacerbated neuronal death, which may lead to increased lesion volume and reduced functional recovery. This iron accentuated ROS production among microglia exposed to $\mathrm{FeSO}_{4}$ \& LPS \& DMSO was significantly reduced following treatment with GSK2795039, suggesting that the presence of superoxide is essential for the mechanism in which iron can accentuate ROS production. NOX4 inhibition was equally effective in reducing iron accentuated ROS production, suggesting that hydrogen peroxide produced by NOX4 contributes to the mechanism of iron accentuated ROS production as well. Overall, the effective reduction of ROS after GSK2795039 and GKT137831 supports our hypothesis that iron undergoes the Fenton, Haber-Weiss reaction utilizing NOX2- and NOX4-derived superoxides and hydrogen peroxide to accentuate ROS production through positive feedback with NOX proteins. It is also important to note that GKT137831 has been described as dual NOX1 and NOX4 inhibitor [37]. However, NOX1 gene expression was not detected in quantitative polymerase chain reaction among BV2 microglia (data not shown), which suggests that NOX1 inhibition is not likely the result of GKT137831 administration in microglia.

The anti-inflammatory effect of iron chelation suggests that iron contributes to a pro-inflammatory response in CNS injury. However, little is known of iron's effect on polarization of microglia. To determine iron's contribution to polarization alterations among microglia, we collected gene expression, FACS, immunocytochemistry, and cytokine/chemokine data. Interestingly, iron alone or in combination with LPS did not significantly alter the gene or protein expression of any polarization or cytokine/chemokine markers assessed in either BV2 or primary microglial cells. This may be due to the overwhelming response to LPS by the cells and therefore a 
ceiling effect is created that additional stimulation by iron cannot surpass or that iron and LPS do not have an additive effect for these outcomes. The observation of a slight decrease in CD86 staining in primary microglia was interesting and suggests that the ceiling effect is not the true cause, however. Further experimentation into the role of iron in microglial cells is warranted.

Despite the lack of an exacerbated polarization response among microglia, an increased neurotoxic effect was observed among neurons exposed to microglia treated with a combination of LPS \& $\mathrm{FeSO}_{4}$. This is likely due to the increased oxidative stress in neurons as a result of increased microglial ROS synthesis. Oxidative stress has been identified as a major contributor to neuronal demise after intracerebral hemorrhagic injury [38]. In 2005, Nakamura et al. reported increased DNA damage after ferrous iron infusion into basal ganglia, which was ameliorated by iron chelation therapy [39]. In addition, this increased oxidative stress phenomena is similar in Parkinsonian neurodegeneration where chronically activated microglia are simultaneously present with increased concentrations of iron [40-42]. This may possibly explain why iron exacerbates CNS injuries and disease.

To further support this conclusion, the observed amelioration of neuronal death by the addition of DFO illustrates the importance of iron mitigation following injury [42, 43]. Multiple studies have found iron chelation therapy can reduce inflammation and lesion volume following CNS injury, potentially via reductions in ROS release [25-27]. We suspect that iron chelation therapy protects microglia from excessive iron and prevents the interaction between free iron and activated microglia.

Both NOX2 and NOX4 inhibition in microglia previously exposed to $\mathrm{FeSO}_{4}$ and LPS significantly increased the presence of neurons in co-culture. This is consistent with recent findings that NOX2 and NOX4 mRNA silencing improved neuronal survivability by preventing apoptosis in a subarachnoid hemorrhage model [6]. In addition, multiple studies suggest improvement of outcomes with various NOX2, NOX4, and non-specific NOX inhibitors, which may be related to their contributions to reduced iron accentuated ROS production after TBI in vivo $[44,45]$.

Due to the heterogeneous nature of primary mixed neuronal cell cultures, we co-cultured microglia with differentiated PC12 neuronal-like cells to isolate the effect of iron accentuated ROS production on neurons. PC12s are neuron-like cells capable of neurite extension and therefore an ideal model to measure the severity of oxidative stress through the apoptotic marker cleaved caspase 3 [46]. Despite PC12's reportedly robust nature, the cultures displayed a significantly increased count of cleaved caspase 3-positive cells after co-culture with microglia previously treated with $\mathrm{FeSO}_{4}$ \& LPS \&
DMSO (Figs. 4 and 5). Inhibition of either NOX2 with GSK2795039 or NOX4 with GKT137831 in microglia prior to co-culture reduced the cellular toxicity among PC12s as evidenced by fewer apoptotic cells. This illustrates the cytotoxic potency of the ROS accentuation by $\mathrm{FeSO}_{4}$ \& LPS and its indirect effect on a cell line reported to have unusually effective antioxidant capabilities.

\section{Conclusions}

The data presented here provide evidence that microglia may passively and indirectly exacerbate neuronal toxicity after CNS damage. These data have been produced in both primary microglial cells and the microglial cell line BV2. To reduce animal use, experiments were primarily conducted in the cell line and confirmed with primary cell line experimentation where possible and most effective. Based on these data, the BV2 cell line appeared to be an acceptable cell for similar responses to primary microglia.

Iron has been identified as an important contributor to oxidative stress in neurodegenerative diseases and seems to be a significant player within TBI pathophysiology as well. Its focus as a primary contributor to oxidative stress is not novel; however, its mitigation in TBI may yield improved therapeutic outcomes by utilizing NOX2 or NOX4 inhibitors. Application of iron chelation or NOX inhibition to CNS injury seems promising [26, 42, 47]. Since effective treatments of CNS injury remain elusive, this may prove to be an effective strategy in the preservation of neurons and ultimately improved functional outcomes.

\section{Additional files}

\begin{abstract}
Additional file 1: Figure S1. BV2 cells express both DMT1 and light \& heavy chain ferritin and take up iron. a) Western blotting of DMT1 revealed increased expression of those groups treated with LPS. b) Gene expression of light and heavy chain ferritin displayed a significant increase in light chain ferritin within $\mathrm{FeSO}_{4}$ groups. Heavy chain ferritin revealed a more robust response to LPS with groups exposed to LPS increasing gene expression. All groups were compared using two-way ANOVA with Tukey post-test. $N=4$ /group. ${ }^{*} p<0.05,{ }^{* *} p<0.01,{ }^{* * *} p<0.0001$. Bars represent mean + /- SEM. c) Quantitation of BV2 microglia incorporation of iron from $\mathrm{FeSO}_{4}$ was done at 6 and $24 \mathrm{~h}$ post-iron exposure. $\mathrm{FeSO}_{4}$ at 10, 25, 50 or $100 \mu \mathrm{M}$ resulted in a concentration dependent increase in iron incorporation that peaked at $100 \mu \mathrm{M}$. Addition of LPS led to a further increase at $24 \mathrm{~h} .{ }^{*} p<0.05$ vs control, ${ }^{* *} p<0.01$ vs control, two-way ANOVA with Dunnett's multiple comparisons post-test. $N=2$ technica replicates. (PDF $2265 \mathrm{~kb}$ )
\end{abstract}

Additional file 2: Figure S2. LDH assay for media from cells treated in the ROS assays in Fig. 1. No significant differences were noted among the treatment groups. $\mathrm{X}$-axis represents $\mathrm{FeSO}_{4}(\mathrm{a}), \mathrm{Fe}\left(\mathrm{NH}_{4}\right)_{2}\left(\mathrm{SO}_{4}\right)_{2}(\mathrm{~b}), \mathrm{NA}_{2} \mathrm{SO}_{4}(\mathrm{c})$. Within the DFO graph, the $X$-axis represents $\mu \mathrm{M}$ concentrations of $\mathrm{FeSO}_{4}$ (d). $\mathrm{LDH}$ release was also assessed in primary microglia with $\mathrm{FeSO}_{4}$ at $100 \mu \mathrm{M}$ (e). All graphs represent an $n=5$. All statistics are one-way ANOVA with Tukey post hoc test. Bars represent mean +/- SEM. (PDF 2065 kb)

Additional file 3: Figure S3. Immunocytochemistry for CD86 in primary microglia shows that iron does not exacerbate M1 polarization. Immunolabeling for CD86 (red) did not show a marked increase with addition of $\mathrm{FeSO}_{4}$. No detectable difference in cell number (as 
qualitatively assessed by DAPI (blue) staining) was noted between groups. Size bar $=50 \mu \mathrm{m}$. (TIF $2223 \mathrm{~kb}$ )

\section{Abbreviations}

CNS: Central nervous system; DFO: Deferoxamine mesylate; DMT1: Divalent metal transporter 1; FACS: Fluorescence-activated cell sorting; FBS: Fetal bovine serum; FER: Ferritin; $H_{2}$ DCFDA: 2',7'-Dichlorodihydrofluorescein diacetate; LPS: Lipopolysaccharide; NOX: NADPH oxidase; ROS: Reactive oxygen species; TBI: Traumatic brain injury

\section{Acknowledgements}

The authors wish to recognize Guzal Khayrullina for technical expertise with flow cytometry and the Proteome Profiler assay and Michael Shaughness for assistance with primary microglia cultures. GKT137831 was donated to the laboratory by Genkyotex, Geneva Switzerland. The authors wish to thank Dr. Joshua Duckworth for research support.

\section{Funding}

This research was completely funded by the Tri Service Nursing Research Program (TSNRP) grant number HU0001-15-1-TS16.

\section{Availability of data and materials}

All data generated or analyzed during this study are included in this published article and its supplementary information files.

\section{Authors' contributions}

YJY conceived of the study; carried out the experiments; performed the statistical analyses for all outcome measures, including the cellular assays, gene expression, and immunoassays; and drafted the manuscript. SB assisted in the design and completion of the cell stimulation model and western blot assays. KEM participated in the design of the gene expression studies and helped to draft the manuscript. EG and BS generated the primary neuron cultures and assisted in data interpretation and analysis. KB participated in the design and coordination of the study and helped to draft the manuscript. All authors read and approved the final manuscript.

\section{Ethics approval and consent to participate}

All animal protocols received approval from the Uniformed Services University of the Health Sciences (USUHS) Institutional Animal Care and Use Committee (IACUC)

\section{Consent for publication}

Not applicable.

\section{Competing interests}

The authors declare that they have no competing interests.

\section{Publisher's Note}

Springer Nature remains neutral with regard to jurisdictional claims in published maps and institutional affiliations.

\section{Author details}

${ }^{1}$ Neuroscience Graduate Program, Uniformed Services University of the Health Sciences, Room C2099, 4301 Jones Bridge Road, Bethesda, MD 20814, USA. ${ }^{2}$ Department of Anatomy, Physiology, and Genetics, Uniformed Services University of the Health Sciences, Room C2099, 4301 Jones Bridge Road, Bethesda, MD 20814, USA. ${ }^{3}$ Department of Anesthesiology and Center for Shock, Trauma, and Anesthesiology Research (STAR), University of Maryland, School of Medicine, 655 W. Baltimore St, Room \#6-015, Baltimore, MD, USA.

Received: 7 September 2018 Accepted: 4 February 2019

\section{Published online: 18 February 2019}

\section{References}

1. Plesnila N. The immune system in traumatic brain injury. Curr Opin Pharmacol. 2016:26:110-7.

2. Loane DJ, Kumar A, Stoica BA, Cabatbat R, Faden Al. Progressive neurodegeneration after experimental brain trauma: association with chronic microglial activation. J Neuropathol Exp Neurol. 2014;73:14-29.
3. Smith DH, Johnson VE, Stewart W. Chronic neuropathologies of single and repetitive TBI: substrates of dementia? Nat Rev Neurol. 2013:9: 211-21.

4. Johnson VE, Stewart JE, Begbie FD, Trojanowski JQ, Smith DH, Stewart W. Inflammation and white matter degeneration persist for years after a single traumatic brain injury. Brain. 2013;136:28-42

5. Lazarus RC, Buonora JE, Jacobowitz DM, Mueller GP. Protein carbonylation after traumatic brain injury: cell specificity, regional susceptibility, and gender differences. Free Radic Biol Med. 2015;78:89-100.

6. Zhang G, Zhang F, Zhang T, Gu J, Li C, Sun Y, Yu P, Zhang Z, Wang Y. Tetramethylpyrazine Nitrone improves neurobehavioral functions and confers neuroprotection on rats with traumatic brain injury. Neurochem Res. 2016:41:2948-57.

7. Khan M, Sakakima H, Dhammu TS, Shunmugavel A, Im YB, Gilg AG, Singh AK, Singh I. S-nitrosoglutathione reduces oxidative injury and promotes mechanisms of neurorepair following traumatic brain injury in rats. J Neuroinflammation. 2011:8:78.

8. Hall ED, Wang JA, Bosken JM, Singh IN. Lipid peroxidation in brain or spinal cord mitochondria after injury. J Bioenerg Biomembr. 2016;48: 169-74

9. Loane DJ, Kumar A. Microglia in the TBI brain: the good, the bad, and the dysregulated. Exp Neurol. 2016;275(Pt 3):316-27.

10. Kroner A, Greenhalgh AD, Zarruk JG, Passos Dos Santos R, Gaestel M, David S. TNF and increased intracellular iron alter macrophage polarization to a detrimental M1 phenotype in the injured spinal cord. Neuron. 2014;83:1098-116.

11. Bridges RA, Berendes $H$, Good RA. A fatal granulomatous disease of childhood: the clinical, pathological, and laboratory features of a new syndrome. AMA J Dis Child. 1959;97:387-408.

12. Iles KE, Forman HJ. Macrophage signaling and respiratory burst. Immunol Res. 2002;26:95-105.

13. Cooney SJ, Bermudez-Sabogal SL, Byrnes KR. Cellular and temporal expression of NADPH oxidase (NOX) isotypes after brain injury. J Neuroinflammation. 2013;10:155.

14. Bedard K, Krause KH. The NOX family of ROS-generating NADPH oxidases: physiology and pathophysiology. Physiol Rev. 2007;87:245-313.

15. Nisenbaum EJ, Novikov DS, Lui YW. The presence and role of iron in mild traumatic brain injury: an imaging perspective. J Neurotrauma. 2014;31:301-7.

16. Chang EF, Claus CP, Vreman HJ, Wong RJ, Noble-Haeusslein LJ. Heme regulation in traumatic brain injury: relevance to the adult and developing brain. J Cereb Blood Flow Metab. 2005:25:1401-17.

17. Ke Y, Qian ZM. Brain iron metabolism: neurobiology and neurochemistry. Prog Neurobiol. 2007;83:149-73.

18. Song $N$, Jiang $H$, Wang J, Xie JX. Divalent metal transporter 1 up-regulation is involved in the 6-hydroxydopamine-induced ferrous iron influx. J Neurosci Res. 2007;85:3118-26.

19. Urrutia P, Aguirre P, Esparza A, Tapia V, Mena NP, Arredondo M, GonzalezBillault C, Nunez MT. Inflammation alters the expression of DMT1, FPN1 and hepcidin, and it causes iron accumulation in central nervous system cells. J Neurochem. 2013:126:541-9.

20. Mehlhase J, Gieche J, Widmer R, Grune T. Ferritin levels in microglia depend upon activation: modulation by reactive oxygen species. Biochim Biophys Acta. 2006;1763:854-9.

21. Braughler JM, Duncan LA, Chase RL. The involvement of iron in lipid peroxidation. Importance of ferric to ferrous ratios in initiation. J Biol Chem. 1986;261:10282-9.

22. Hall ED, Andrus PK, Yonkers PA, Smith SL, Zhang JR, Taylor BM, Sun FF. Generation and detection of hydroxyl radical following experimental head injury. Ann N Y Acad Sci. 1994;738:15-24.

23. Globus MY, Alonso O, Dietrich WD, Busto R, Ginsberg MD. Glutamate release and free radical production following brain injury: effects of posttraumatic hypothermia. J Neurochem. 1995;65:1704-11.

24. Nishio S, Yunoki M, Noguchi Y, Kawauchi M, Asari S, Ohmoto T. Detection of lipid peroxidation and hydroxyl radicals in brain contusion of rats. Acta Neurochir Suppl. 1997:70:84-6.

25. Li Y, Pan K, Chen L, Ning JL, Li X, Yang T, Terrando N, Gu J, Tao G. Deferoxamine regulates neuroinflammation and iron homeostasis in a mouse model of postoperative cognitive dysfunction. J Neuroinflammation. 2016;13:268

26. Zhang L, Hu R, Li M, Li F, Meng H, Zhu G, Lin J, Feng H. Deferoxamine attenuates iron-induced long-term neurotoxicity in rats with traumatic brain injury. Neurol Sci. 2013;34:639-45. 
27. Haleagrahara N, Siew CJ, Ponnusamy K. Effect of quercetin and desferrioxamine on 6-hydroxydopamine (6-OHDA) induced neurotoxicity in striatum of rats. J Toxicol Sci. 2013;38:25-33.

28. Das A, Kim SH, Arifuzzaman S, Yoon T, Chai JC, Lee YS, Park KS, Jung KH, Chai YG. Transcriptome sequencing reveals that LPS-triggered transcriptional responses in established microglia BV2 cell lines are poorly representative of primary microglia. J Neuroinflammation. 2016;13:182.

29. Chenais B, Morjani H, Drapier JC. Impact of endogenous nitric oxide on microglial cell energy metabolism and labile iron pool. J Neurochem. 2002;81:615-23.

30. Ghosh M, Xu Y, Pearse DD. Cyclic AMP is a key regulator of M1 to M2a phenotypic conversion of microglia in the presence of Th2 cytokines. J Neuroinflammation. 2016;13:9.

31. Stansley B, Post J, Hensley K. A comparative review of cell culture systems for the study of microglial biology in Alzheimer's disease. J Neuroinflammation. 2012;9:115

32. Blasi E, Barluzzi R, Bocchini V, Mazzolla R, Bistoni F. Immortalization of murine microglial cells by a v-raf/v-myc carrying retrovirus. J Neuroimmunol. 1990;27:229-37.

33. Tamashiro TT, Dalgard CL, Byrnes KR. Primary microglia isolation from mixed glial cell cultures of neonatal rat brain tissue. J Vis Exp. 2012;(66):e3814.

34. Yakovlev AG, Ota K, Wang G, Movsesyan V, Bao WL, Yoshihara K, Faden Al. Differential expression of apoptotic protease-activating factor- 1 and caspase-3 genes and susceptibility to apoptosis during brain development and after traumatic brain injury. J Neurosci. 2001;21:7439-46.

35. Das KP, Freudenrich TM, Mundy WR. Assessment of PC12 cell differentiation and neurite growth: a comparison of morphological and neurochemical measures. Neurotoxicol Teratol. 2004;26:397-406.

36. von Leden RE, Yauger YJ, Khayrullina G, Byrnes KR. Central nervous system injury and nicotinamide adenine dinucleotide phosphate oxidase: oxidative stress and therapeutic targets. J Neurotrauma. 2017; 34:755-64.

37. Deliyanti D, Wilkinson-Berka JL. Inhibition of NOX1/4 with GKT137831: a potential novel treatment to attenuate neuroglial cell inflammation in the retina. J Neuroinflammation. 2015:12:136.

38. Wagner KR, Packard BA, Hall CL, Smulian AG, Linke MJ, De Courten-Myers GM, Packard LM, Hall NC. Protein oxidation and heme oxygenase-1 induction in porcine white matter following intracerebral infusions of whole blood or plasma. Dev Neurosci. 2002;24:154-60.

39. Nakamura T, Keep RF, Hua Y, Hoff JT, Xi G. Oxidative DNA injury after experimental intracerebral hemorrhage. Brain Res. 2005;1039:30-6.

40. Zucca FA, Segura-Aguilar J, Ferrari E, Munoz P, Paris I, Sulzer D, Sarna T, Casella L, Zecca L. Interactions of iron, dopamine and neuromelanin pathways in brain aging and Parkinson's disease. Prog Neurobiol. 2017;155: 96-119.

41. Zhu Y, Wang B, Tao K, Yang H, Wang Y, Zhou T, Yang Y, Yuan L, Liu X, Duan $Y$. Iron accumulation and microglia activation contribute to substantia nigra hyperechogenicity in the 6-OHDA-induced rat model of Parkinson's disease. Parkinsonism Relat Disord. 2017;36:76-82.

42. Aguirre P, Mena NP, Carrasco CM, Munoz Y, Perez-Henriquez P, Morales RA, Cassels BK, Mendez-Galvez C, Garcia-Beltran O, Gonzalez-Billault C, Nunez MT. Iron chelators and antioxidants regenerate neuritic tree and nigrostriatal fibers of MPP+/MPTP-lesioned dopaminergic neurons. PLoS One. 2015;10:e0144848.

43. Sun YM, Wang YT, Jiang L, Xue MZ. The effects of deferoxamine on inhibition for microglia activation and protection of secondary nerve injury after intracerebral hemorrhage in rats. Pak J Pharm Sci. 2016;29:1087-93.

44. Zhang QG, Laird MD, Han D, Nguyen K, Scott E, Dong Y, Dhandapani KM Brann DW. Critical role of NADPH oxidase in neuronal oxidative damage and microglia activation following traumatic brain injury. PLoS One. 2012;7:e34504.

45. Wang $\mathrm{Q}$, Chu CH, Oyarzabal E, Jiang L, Chen SH, Wilson B, Oian L, Hong JS. Subpicomolar diphenyleneiodonium inhibits microglial NADPH oxidase with high specificity and shows great potential as a therapeutic agent for neurodegenerative diseases. Glia. 2014;62:2034-43.

46. Piga R, Saito Y, Yoshida Y, Niki E. Cytotoxic effects of various stressors on PC12 cells: involvement of oxidative stress and effect of antioxidants. Neurotoxicology. 2007;28:67-75.

47. You LH, Li F, Wang L, Zhao SE, Wang SM, Zhang LL, Zhang LH, Duan XL, Yu $P$, Chang YZ. Brain iron accumulation exacerbates the pathogenesis of MPTP-induced Parkinson's disease. Neuroscience. 2015;284:234-46.

\section{Ready to submit your research? Choose BMC and benefit from:}

- fast, convenient online submission

- thorough peer review by experienced researchers in your field

- rapid publication on acceptance

- support for research data, including large and complex data types

- gold Open Access which fosters wider collaboration and increased citations

- maximum visibility for your research: over $100 \mathrm{M}$ website views per year

At $\mathrm{BMC}$, research is always in progress.

Learn more biomedcentral.com/submissions 